\title{
IRX2-mediated upregulation of MMP-9 and VEGF in a PI3K/AKT-dependent manner
}

\author{
TIELONG LIU ${ }^{1 *}$, WEIWEI ZHOU ${ }^{2 *}$, BING CAI $^{3 *}$, JIANJUN CHU $^{4}$, GUODONG SHI $^{1}$, \\ HONGLIN TENG ${ }^{5}$, JIAN XU ${ }^{6}$, JIANRU XIAO ${ }^{1}$ and YAN WANG $^{7}$
}

\author{
Departments of ${ }^{1}$ Orthopedics and ${ }^{2}$ Radiology, Changzheng Hospital, Second Military Medical University, Shanghai 200003; \\ ${ }^{3}$ Department of Orthopedics, Ningbo Development Zone Center Hospital, Ningbo, Zhejiang 315800; \\ ${ }^{4}$ Department of Orthopedics, First People's Hospital of Hefei, Hefei, Anhui 230061; ${ }^{5}$ Department of Orthopedics, \\ First Affiliated Hospital, Wenzhou Medical University, Wenzhou, Zhejiang 325000; ${ }^{6}$ Department of Orthopedics, \\ The Workers Hospital of Suqian City, Suqian, Jiangsu 223800; ${ }^{7}$ Department of Orthopedics, \\ Chinese People's Liberation Army General Hospital, Beijing 100853, P.R. China
}

Received July 3, 2014; Accepted April 10, 2015

DOI: $10.3892 / \mathrm{mmr} .2015 .3915$

\begin{abstract}
Osteosarcoma (OS) is the most frequent type of primitive malignant bone tumor with a poor prognosis due to distant metastasis. Our previous studies have demonstrated that IRX2 is overexpressed and is important in cell proliferation and invasion. However, the molecular mechanisms underlying the IRX2-dependent regulation of OS progression remains to be elucidated. In the present study, the effects of IRX 2 on the upregulation of MMP2 and VEGF in OS were determined by western blotting, and the underlying molecular mechanisms were elucidated. These findings provided data suggesting that IRX2 modulates the expression levels of MMP2 and VEGF in an AKT-dependent manner. The overexpression of IRX2 promoted the activation of PI3K/Akt and increased the proliferation and invasiveness of the OS cell lines as shown by CCK8 and invasion assays. Notably, interruption of the AKT pathway by treatment with LY294002, a specific PI3K inhibitor, attenuated IRX2-induced cell proliferation and invasive ability, and the upregulation of MMP2 and VEGF. The results of the present study suggested that inhibition of the IRX2-mediated AKT signaling pathway may be a suitable therapeutic target for the treatment of OS.
\end{abstract}

Correspondence to: Miss Jianru Xiao, Department of Orthopedics, Changzheng Hospital, Second Military Medical University, 28 Fuxing Road, Shanghai 200003, P.R. China

E-mail: jianru321xiao@163.com

Professor Yan Wang, Department of Orthopedics, Chinese People's Liberation Army General Hospital, 28 Fuxing Road, Beijing 100853, P.R. China

E-mail: yanwangarmy@163.com

*Contributed equally

Key words: osteosarcoma, IRX2, cell growth, cell invasion, AKT

\section{Introduction}

Osteosarcoma (OS) is the most common primary malignancy, predominantly arising from metaphysis of the long bones of adolescents and young adults (1). Almost $80 \%$ of the cases of OS occur at the rapidly growing sites of longitudinal bones, including the distal femur, proximal tibia and proximal humerus (1-3). Despite current treatments, combining chemotherapy, surgery and often radiotherapy, patients with recurrent or metastatic OS exhibit a poor prognosis, with a 50-60\% 5-year-survival rate worldwide (1). Elucidation of the fundamental molecular mechanisms underlying the initiation, drug resistance and development of metastasis is important and urgent for the development of the next generation of anticancer therapeutics for OS $(2,4)$.

Our previous study demonstrated that the mRNA expression of IRX2 is upregulated in OS and acts as an oncogene (5). IRX2 is a member of the Iroquois homeobox gene family and its expression has been observed to increase in several types of cancer, including acute lymphoblastic leukemia, breast cancer and soft tissue sarcoma (6-8). Furthermore, a previous study revealed that the DNA methylation levels of IRX2 are associated with the aggressiveness of tumor development and the outcome of patients with lung cancer (9).

The PI3K/Akt pathway is a key regulator of several physiological and pathological conditions. Previous studies have revealed that the activation of Akt activates signaling cascades, which contribute to sustaining tumor outgrowth and metastasis, including the mTOR and MAPK signaling pathways (10-12). The activation of Akt has been demonstrated to induce the transcription of a set of target genes, which ultimately leads to the regulation of cell growth, motility and apoptosis in OS (13-16). In addition, the inactivation of Akt by other genes or by a specific PI3K inhibitor induces G0/G1 cell cycle arrest, inhibits OS cell proliferation, migration and invasion, and promotes apoptosis $(15,17)$. However, whether the activation of Akt is important in IRX2-induced cell growth and invasion remains to be elucidated. 
In the present study, the regulatory mechanism by which IRX2 potentiates proliferation and invasion through regulating the PI3K/AKT signal pathway was investigated, which may provide a potential therapeutic target for better management of OS.

\section{Materials and methods}

Tumor specimens. A total of 68 paired fresh and frozen paired OS tumor tissues and adjacent normal tissues were collected from Changzheng Hospital (Shanghai, China) between 2004 and 2009. Patients were diagnosed with OS based on histopathological evaluation. The numbers of patients staged I, II and III were 1, 36 and 31, respectively. The details of the patients were as follows: Age, 7-45 years; gender, 29 female and 39 male; tumor location, 38 femur, 16 tibia, 9 humerus, 5 others. The patients had not received chemotherapy or radiotherapy prior to surgery. The present study was performed with the approval of the Medical Ethical Committee of the Second Military Medical University (Shanghai, China). Written informed consent was obtained from the patient/patient's family. All tissue samples were flash-frozen in liquid nitrogen immediately following collection, and stored at $-80^{\circ} \mathrm{C}$ until use.

Plasmid construction. The coding sequence of human IRX2 (NM_033267.4; NCBI Reference Sequence, OriGene Technologies, Rockville, MD, USA) was amplified by performing polymerasechain reaction (PCR) using the forward primer, 5'-GGATCCATGTCCTACCCGCAGGGC-3', which introduced a $B a m \mathrm{HI}$ site, and the reverse primer, 5'-GAATTCCTATAGGTAGGGCTGGACGC-3' (Sangon Biotech Co., Ltd., Shanghai, China), which introduced an EcoRI site. The PCR conditions were as follows: Initial denaturation at $98^{\circ} \mathrm{C}$ for $2 \mathrm{~min}$, followed by 30 cycles of denaturation at $98^{\circ} \mathrm{C}$ for $15 \mathrm{sec}$, primer annealing at $55^{\circ} \mathrm{C}$ for $15 \mathrm{sec}$, and a primer extension step at $72^{\circ} \mathrm{C}$ for $2 \mathrm{~min}$, using high-fidelity polymerase PrimeStar (Takara Bio Inc., Otsu, Japan). The products of the PCR were then cloned into the BamH1 and EcoRI sites of the pCMV Tag 2B constitutive mammalian expression vector (Stratagene, La Jolla, CA, USA), according to the manufacturer's instructions. The sequence of IRX2 was confirmed by sequencing (Sangon Biotech Co., Ltd.). This vector was termed pCMV-IRX2 (IRX2). The pCMV Tag 2B empty vector (termed vector) was used as a negative control under similar conditions. The overexpression efficiency was assessed $48 \mathrm{~h}$ following transfection by measuring the protein expression levels in the cell lysates using western blot analysis.

Cell culture and transfection. The human MG63 and SaOS2 OS cell lines were purchased from American Type Culture Collection (Rockville, MD, USA) and cultured in Dulbecco's modified Eagle's medium, supplemented with $10 \%$ fetal calf serum, $100 \mathrm{U} / \mathrm{ml}$ penicillin and $100 \mu \mathrm{g} / \mathrm{ml}$ streptomycin (all Invitrogen Life Technologies, Carlsbad, CA, USA), in a $37^{\circ} \mathrm{C}$ humidified and $5 \% \mathrm{CO}_{2}$ incubator. For the transfection experiments, plasmids overexpressing IRX2, the coding sequence of human IRX2, without the stop codon were constructed and were inserted into the pcDNA3.1 vector (Invitrogen Life
Technologies). A total of $2 \times 10^{5}$ cells/well were plated into a 24-well plate $24 \mathrm{~h}$ prior to transfection, and were grown to $30-50 \%$ confluence. Effective transfection reagent (Qiagen Inc., Hilden, Germany) was used to perform transfection and either $2.0 \mu \mathrm{g}$ pCMV-IRX2 vector or $2.0 \mu \mathrm{g}$ pCMV Tag 2B empty vector were used, according to the manufacturer's instructions. Dimethyl sulfoxide and $10 \mu \mathrm{M}$ LY294002 (Sigma-Aldrich) was used to treat the cells for $2 \mathrm{~h}$.

Western blotting. As previously described (5), the total proteins were extracted from the cells and tissue samples using radioimmunoprecipitation buffer (Cell Signaling Technology, Inc., Danvers, MA, USA), containing Protease Inhibitors Cocktail tablets (Roche Diagnostics, Mannheim, Germany). The proteins were quantified using a bicinchoninic acid assay (Sangon Biotech, Co, Ltd.), and $50 \mu \mathrm{g}$ protein from each sample was separated on $10 \%$ SDS-polyacrylamide gels and transferred onto polyvinylidene fluoride membranes (Bio-Rad Laboratories, Inc., Hercules, CA, USA). The membranes were blocked using $6 \%$ not-fat milk in Tris-buffered saline, containing $1 \%$ Tween-20. The membranes were then incubated with the following antibodies: Mouse polyclonal anti-IRX2 (ab72975) and rabbit monoclonal anti-VEGF (ab52917) antibodies (1:500; Abcam, Cambridge, CA, USA), rabbit polyclonal anti-AKT (\#9272), rabbit polyclonal anti-phosphorylated (p)-AKT (\#9271) and rabbit polyclonal anti-MMP-9 (\#3852) antibodies (1:1,000; Cell Signaling Technology, Inc.), and rabbit polyclonal anti-actin antibody (CW0097; 1:5,000; Cwbiotech, Beijing, China), which was used as an internal control. Membranes were incubated with horseradish peroxidase (HRP)-labeled anti-rabbit secondary antibody (CW0103; 1:3000; Cwbiotech) at room temperate for $1 \mathrm{~h}$. The proteins were detected using an Image Quant $^{\mathrm{TM}}$ LAS-4000 (Fujifilm, Tokyo, China) and the chemiluminescence was directly analyzed and quantified using Image Quant TL version 2005 software (GE Healthcare Bio-Sciences, Pittsburgh, PA, USA).

Cell proliferation assay. Cell viability was assessed using a Cell Counting kit-8 (CCK8; Dojindo Laboratories, Kumamoto, Japan), according to the manufacturer's instructions. CCK8 is more sensitive, compared with the 3-(4,5-dimethylthiazol-2-yl)-2,5-diphenyltetrazolium bromide assay. The MG63-IRX2 and SaOS2-IRX2 cells were treated with $10 \mu \mathrm{lCCK} 8$ at $37^{\circ} \mathrm{C}$ for $1 \mathrm{~h}$. The absorbance was then measured at $450 \mathrm{~nm}$ using a microplate reader (Model 680; Bio-Rad Laboratories, Inc.) .

Invasion assay. The invasive activity of the pCMV-IRX2 transfected human OS cells was assessed using BD Falcon ${ }^{\mathrm{TM}}$ Cell culture inserts coated with BD Matrigel ${ }^{\mathrm{TM}}$ Basement Membrane Matrix (BD Biosciences, Bedford, MA, USA). Briefly, the transfected MG63 and SaOS2 cells were resuspended in serum free medium and seeded into the upper chamber of the assay system. The lower wells of the system were filled with complete growth medium. Following incubation for $48 \mathrm{~h}$, the invaded cells were washed twice with ice-cold phosphate-buffered saline, fixed with $4 \%$ paraformaldehyde (Sangon Biotech Co., Ltd.) for $15 \mathrm{~min}$ and stained with methyl violet $(0.01 \%$ v/v; Guidechem, Shanghai, 
A

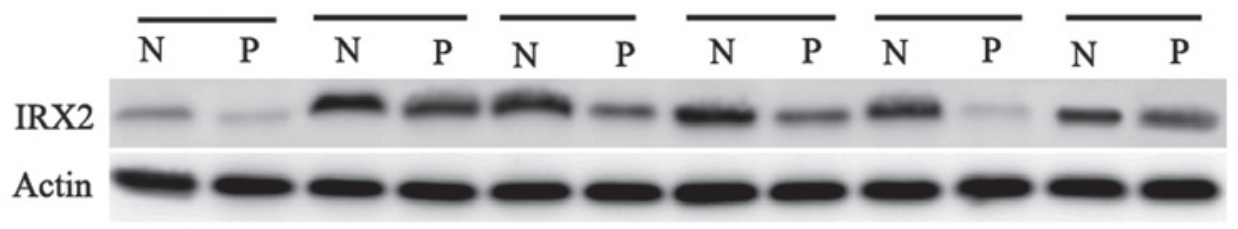

B

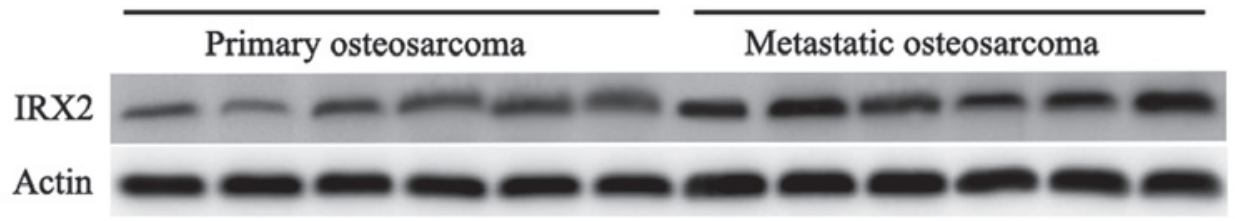

Figure 1. Increased expression of IRX2 was observed in osteosarcoma tissue samples, determined using western blot analysis. (A) Results of western blot analysis for the expression of IRX2 in eight paired osteosarcoma and corresponding normal tissue samples. (B) Proteins were extracted from primary and metastatic tissue samples. $\beta$-actin was used as a loading control. N, normal; P, paired osteosarcoma.

China) for $30 \mathrm{~min}$. The numbers of invaded cells were then counted in more than five randomly selected fields under a light microscope (IX73-F22FL/PH; Olympus America, Inc., Melville, NY, USA).

Statistical analysis. The data are expressed as the mean \pm standard deviation and were analyzed using Student's t-test. Statistical analyses were two-sided and were performed using SPSS 15 software (SPSS, Inc., Chicago, IL, USA). $\mathrm{P}<0.05$ was considered to indicate a statistically significant difference.

\section{Results}

Expression of IRX2 is significantly upregulated in OS tissue. Our previous study reported that the mRNA expression levels of IRX2 were significantly higher in OS tissue compared with adjacent non-tumor tissue (5). Due to a lack of appropriate antibody against IRX2, semiquantitative analysis of a tissue array containing small sections of benign and malignant tumor samples were not assessed using immunohistochemistry. To further assess the expression of IRX2, western blotting was performed to determine the protein expression of IRX2. As shown in Fig. 1A, the protein expression of IRX2 was assessed in individual normal and malignant tissue samples on the array, and marked overexpression was observed in the malignant tissue samples, compared with the normal tissue samples. In addition, the expression levels of IRX2 were compared between primary OS without primary metastasis and OS with metastasis (Fig.1B). The expression of IRX2 was more marked in primary OS with metastasis, compared with primary OS without metastasis. These results demonstrated that the expression of IRX2 increased in OS, which suggested that IRX2 may be involved in the progression of OS.

IRX2 promotes OS cell growth and invasion in vitro. The effect of IRX 2 on the proliferation and migration of OS cancer cells was assessed by knocking down IRX2 (5). To further assess the role of IRX2 in OS cancer cells, a mammalian expression vector was used to force the expression of IRX2. It was observed that IRX2 cDNA transfection increased the mRNA and protein expression levels of IRX2 in the MG63 and SaOS2 cells (Fig. 2A and B). To confirm whether IRX2 regulated OS cell growth, an MTT assay was performed, the results of which demonstrated that overexpression of IRX2 significantly promoted cell growth in the two OS cell lines (Fig. 2C). The invasion assays demonstrated that the forced expression of IRX2 led to an increase in invasive ability of the cells transfected with IRX2, compared with the cells transfected with the control vector (Fig. 2D). These results further confirmed IRX2 as an oncogene and that it was important in the progression of OS.

IRX2 upregulates the expression of $p-A K T / M M P-9$ and VEGF signaling pathways. Our previous study demonstrated that inhibition of the expression of IRX2 inactivated the PI3K/Akt signaling pathway and reduced the expression of MMP-9 (5). The present study proceeded to determine whether the overexpression of IRX2 activated the PI3K/Akt signaling pathway and increased the expression of MMP-9. In agreement with the results observed from knocking out IRX2, overexpression of IRX2 in the MG63 and SaOS2 cells led to a significant increase in the expression levels of p-AKT and MMP-9 (Fig. 3). In addition, VEGF, which is an important mediator of vascularization and the expression of which is associated with increased aggressive behavior $(18,19)$, was markedly upregulated in the cells transfected with IRX2 (Fig. 3). These finding suggested a mechanism, whereby the PI3K/Akt signaling pathway may be a required medium in IRX2-induced cell proliferation and invasion in OS cells.

PI3K/AKT is required for IRX2-enhanced cell proliferation and invasion, and for the activation of MMP-9 and VEGF in OS cells. Activation of the PI3K/AKT signaling pathway appears to be involved in various aspects of tumor development, including cell proliferation, differentiation, migration, invasion, apoptosis and angiogenesis, in several types of cancer $(20,21)$. Previous studies have demonstrated that the $\mathrm{PI} 3 \mathrm{~K} / \mathrm{AKT}$ signaling pathway is important in the proliferation and metastasis of OS cells by regulating the expression levels of MMP-9 and VEGF $(22,23)$. To examine whether IRX2-induced cell proliferation and invasion is dependent on increased PI3K/AKT signaling pathway activity, the PI3K/AKT pathway was inhibited using Ly294002, a PI3K inhibitor. Inhibition of PI3K markedly reduced the invasive 
A

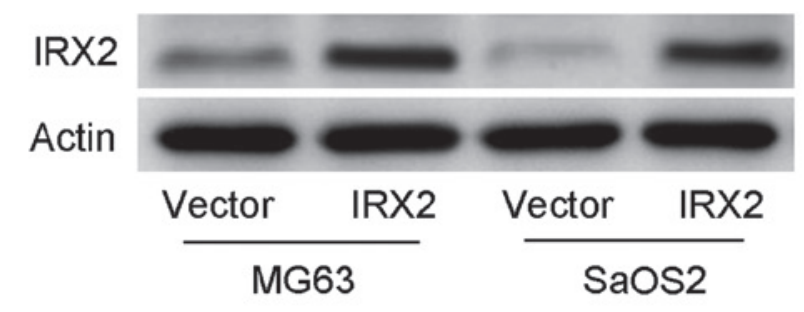

$\mathbf{C}$

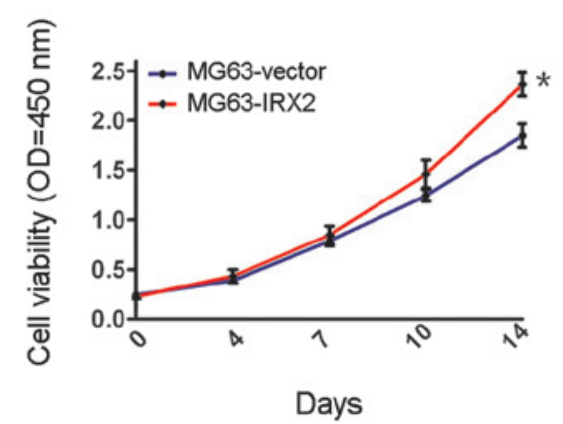

D

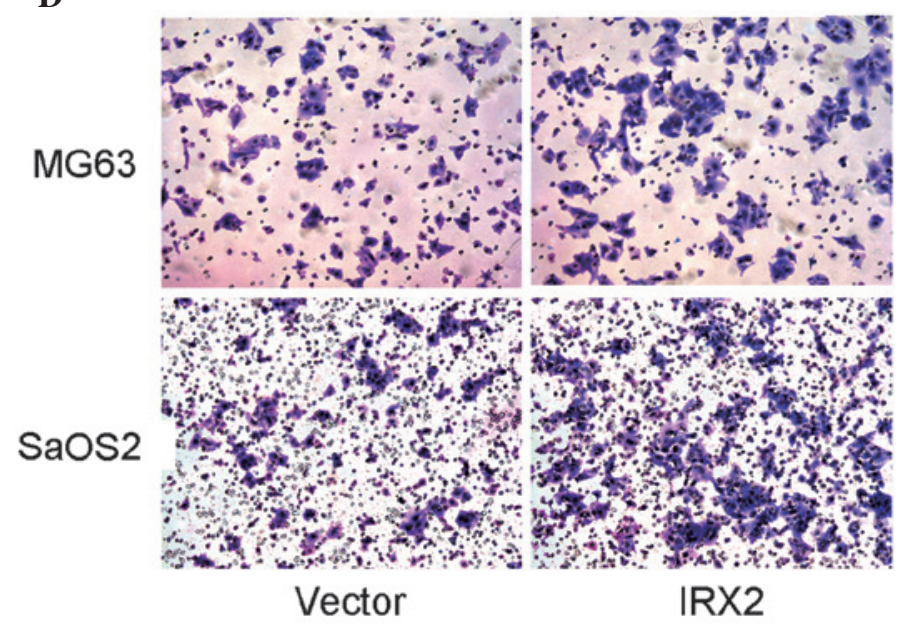

B
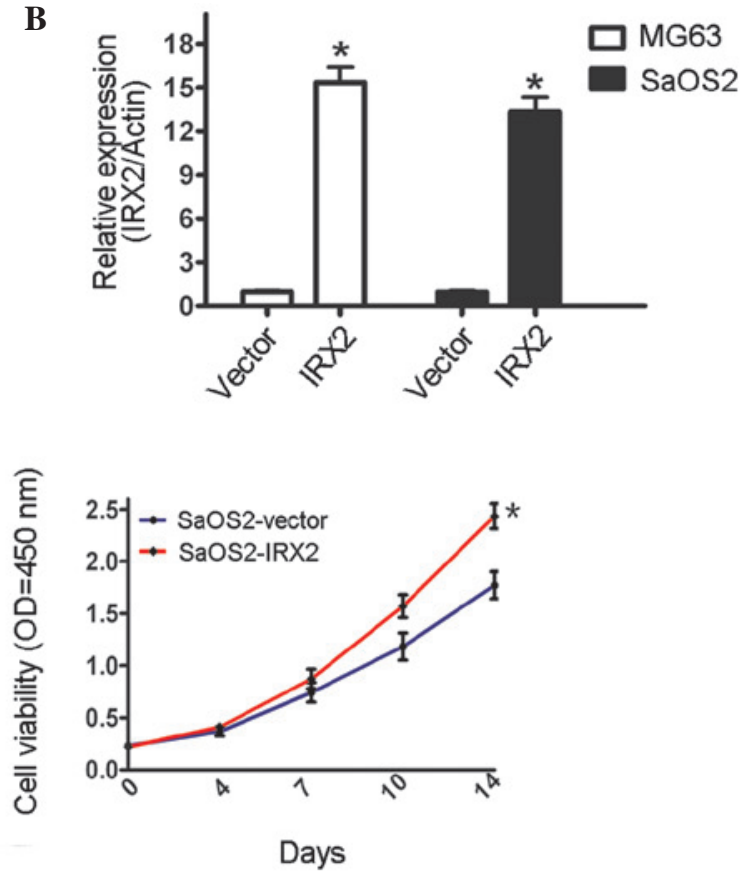

Figure 2. Increased expression of IRX2 promotes osteosarcoma cell growth and invasion in vitro. (A and B) Protein and mRNA expression levels of IRX2 were determined in MG63 and SaOS2 osteosarcoma cells transfected with IRX2 or a control vector. (C) An MTT assay was performed to assess the cell growth of MG63 and SaOS2 cells transfected with IRX2 or the control vector. (D) Representative images of the invasion assay of IRX2-transfected osteosarcoma cells. The MG63 and SaOS2 cells were transfected with IRX2 and exhibited increased invasive activity, compared with the control vector-transfected cells (magnification, x100). Quantitative analysis of cell invasion was assessed following $48 \mathrm{~h}$ of chemo-attraction. Values represent the mean \pm standard deviation of triplicates in one representative experiment. " $\mathrm{P}<0.05$, compared with the control vector. OD, optical density.

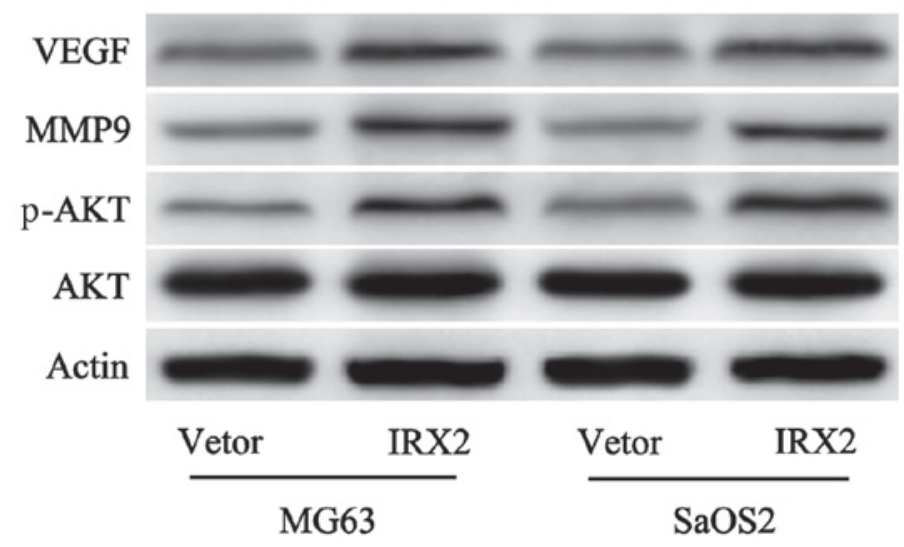

Figure 3. IRX2 induces the phosphorylation of Akt and increases the expression levels of MMP-9 and VEGF in osteosarcoma cells. Western blot analysis of the protein expression of VEGF, MMP-9, p-AKT and AKT in the indicated cells. $\beta$-actin was used as an internal control. p-, phosphorylated. 
A

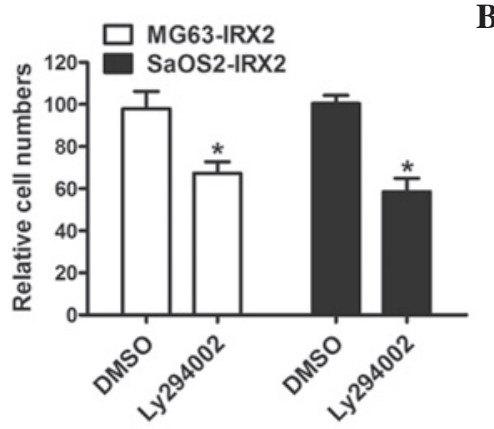

B

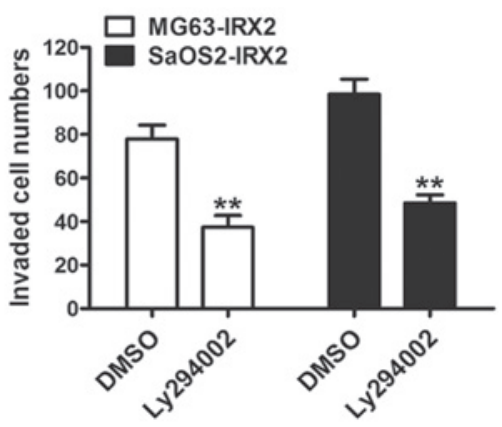

C

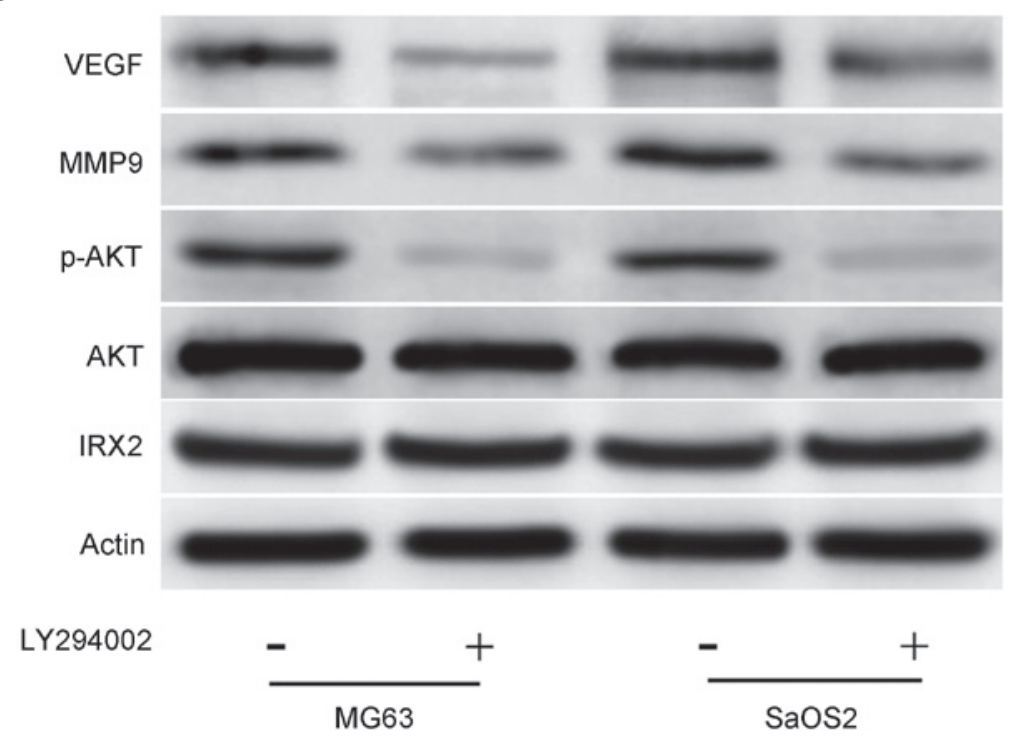

Figure 4. PI3K/AKT are involved in IRX2-induced cell proliferation, invasion and upregulation of MMP-9 and VEGF. (A) Effects of Ly294002 on cell proliferation were measured using an MTT assay in MG63 and SaOS2 cells transfected with IRX2. (B) Effects of Ly294002 on cell invasion were assessed using an invasion assay in MG63 and SaOS2 cells transfected with IRX2. "P<0.05 and ${ }^{* *} \mathrm{P}<0.01$, vs. DMSO control. (C) MG63 and SaOS2 cells transfected with IRX2 were treated with or without Ly294002, and the expression levels of VEGF, MMP-9, p-Akt and AKT were detected using western blot analysis. p-, phosphorylated. DMSO, dimethyl sulfoxide.

abilities of the IRX-transfected MG63 and SaOS2 cells, compared with the control vector-transfected cells (Fig. 4A). Furthermore, IRX2-mediated OS cell invasion was markedly inhibited by Ly294002 (Fig. 4B). To further investigate whether the activation of the PI3K/AKT pathway is involved in IRX2-mediated upregulation of the expression levels of MMP-9 and VEGF, western blotting was performed. The results revealed that the protein expression levels of MMP-9 and VEGF were significantly decreased (Fig. 4C). These data suggested that activation of the PI3K/Akt survival pathway contributed to IRX2-induced growth and migration in the OS cells.

\section{Discussion}

OS is the most common primary malignant tumor of bone, generally following an aggressive clinical course, and presents a major therapeutic challenge (24). Despite progress in understanding the molecular biology of OS, elucidation of the underlying mechanisms associated with the development of OS is important for effective treatment strategies.

The present study established an IRX2-overexpression model and observed that IRX2 promoted cell growth and invasion in vitro. There were several lines of evidence to support this. The expression of IRX2 was first confirmed to be upregulated in OS tissue, particularly in patients with tumor metastasis. Secondly, overexpression of IRX2 was observed to promote the viability and invasion of the OS cells. Thirdly, IRX2 activated the PI3K/Akt signaling pathway and induced the upregulation of the expression levels of MMP-9 and VEGF. In addition, the present study provided evidence demonstrating that activation of the PI3K/Akt signaling pathway was involved in IRX2-induced cell viability and invasion, and in upregulation of MMP-9 and VEGF in the OS cells. These results indicated for the first time, to the best of our knowledge, that IRX2 promoted OS cell growth and invasion through the PI3K/Akt signaling-mediated activation of MMP-9 and VEGF.

Although a number of previous studies have indicated that IRX2 genes are usually amplified in cancer progression (6-8), the detail mechanisms involved in tumor progression remained to be elucidated. Our previous studies demonstrated that IRX2 silencing significantly reduces the proliferation and inhibits the invasiveness of U2OS and SaOS2 OS cells (5). The PI3K/Akt signaling pathway is involved in development and tumorigenesis by regulating several aspects of cell 
proliferation and invasiveness $(12,13,21)$. Angiogenesis is required to sustain primary tumor growth and metastasis (25). The present study revealed that IRX2 transfection increased the phosphorylation of AKT and upregulation of MMP-9 and VEGF. To confirm that the PI3K/AKT pathway was involved in the IRX2-mediated cell proliferation and invasion of the OS cells, the PI3K/AKT pathway was inhibited using Ly294002, a PI3K inhibitor. The results demonstrated that the phosphorylation of AKT was inhibited by the specific inhibitors independently. IRX2-induced proliferation and invasion were reversed and the protein expression levels of MMP-9 and VEGF were significantly reduced by Ly294002, suggesting that IRX2 affected cell proliferation and invasion, and the expression levels of MMP-9 and VEGF via the PI3K/AKT signaling pathways.

In conclusion, the results of the present study provided further evidence that the expression of IRX2 was frequently increased in OS tissue samples, and that a novel molecular mechanism responsible for the IRX2-induced OS cell growth involved the PI3K/AKT signaling-mediated production of MMP-9 and VEGF.

\section{References}

1. Picci P, Mercuri M, Ferrari S, et al: Survival in high-grade osteosarcoma: Improvement over 21 years at a single institution. Ann Oncol 21: 1366-1373, 2010.

2. Broadhead ML, Clark JC, Myers DE, Dass CR and Choong PF: The molecular pathogenesis of osteosarcoma: A review. Sarcoma 2011: 959248, 2011.

3. Cesari M, Alberghini M, Vanel D, et al: Periosteal osteosarcoma: A single-institution experience. Cancer 117: 1731-1735, 2011.

4. Thomas DM: Wnts, bone and cancer. J Pathol 220: 1-4, 2010

5. Liu T, Zhou W, Zhang F, et al: Knockdown of IRX2 inhibits osteosarcoma cell proliferation and invasion by the AKT/MMP9 signaling pathway. Mol Med Rep 10: 169-174, 2014.

6. Kadota M, Sato M, Duncan B, et al: Identification of novel gene amplifications in breast cancer and coexistence of gene amplification with an activating mutation of PIK3CA. Cancer Res 69 7357-7365, 2009.

7. Adamowicz M, Radlwimmer B, Rieker RJ, et al: Frequent amplifications and abundant expression of TRIO, NKD2 and IRX2 in soft tissue sarcomas. Genes Chromosomes Cancer 45: 829-838, 2006.

8. Kang H, Wilson CS, Harvey RC, et al: Gene expression profiles predictive of outcome and age in infant acute lymphoblastic leukemia: A Children's Oncology Group study. Blood 119 1872-1881, 2012.
9. Sato T, Arai E, Kohno T, et al: Epigenetic clustering of lung adenocarcinomas based on DNA methylation profiles in adjacent lung tissue: Its correlation with smoking history and chronic obstructive pulmonary disease. Int J Cancer 135: 319-334, 2014.

10. Francipane MG and Lagasse E: mTOR pathway in colorectal cancer: An update. Oncotarget 5: 49-66, 2014.

11. Pandurangan AK and Esa NM: Signal transducer and activator of transcription 3-a promising target in colitis-associated cancer. Asian Pac J Cancer Prev 15: 551-560, 2014

12. Martini M, De Santis MC, Braccini L, Gulluni F and Hirsch E: PI3K/AKT signaling pathway and cancer: an updated review. Ann Med 46: 372-383, 2014.

13. Vivanco I and Sawyers CL: The phosphatidylinositol 3-Kinase AKT pathway in human cancer. Nat Rev Cancer 2: 489-501, 2002.

14. Guo YS, Zhao R, Ma J, et al: $\beta$ ig-h3 promotes human osteosarcoma cells metastasis by interacting with integrin alpha2betal and activating PI3K signaling pathway: PLoS One 9: e90220, 2014.

15. Gong C, Liao H, Wang J, et al: LY294002 induces G0/G1 cell cycle arrest and apoptosis of cancer stem-like cells from human osteosarcoma via down-regulation of PI3K activity. Asian Pac J Cancer Prev 13: 3103-3107, 2012.

16. Zhang A, He S, Sun X, et al: Wnt5a promotes migration of human osteosarcoma cells by triggering a phosphatidylinositol-3 kinase/Akt signals. Cancer Cell Int 14: 15, 2014.

17. Zhao H, Li M, Li L, et al: MiR-133b is down-regulated in human osteosarcoma and inhibits osteosarcoma cells proliferation, migration and invasion and promotes apoptosis. PLoS One 8: e83571, 2013

18. Kang J, Rychahou PG, Ishola TA, et al: N-myc is a novel regulator of PI3K-mediated VEGF expression in neuroblastoma. Oncogene 27: 3999-4007, 2008.

19. Garcia V, Garcia JM, Silva J, et al: Levels of VEGF-A mRNA in plasma from patients with colorectal carcinoma as possible surrogate marker of angiogenesis. J Cancer Res Clin Oncol 134: 1165-1171, 2008.

20. Dillon RL and Muller WJ: Distinct biological roles for the akt family in mammary tumor progression. Cancer Res 70 : 4260-4264, 2010.

21. Hassan B, Akcakanat A, Holder AM and Meric-Bernstam F: Targeting the PI3-kinase/Akt/mTOR signaling pathway. Surg Oncol Clin N Am 22: 641-664, 2013.

22. Liao CL, Lai KC, Huang AC, et al: Gallic acid inhibits migration and invasion in human osteosarcoma U-2 OS cells through suppressing the matrix metalloproteinase-2/-9, protein kinase $\mathrm{B}$ (PKB) and PKC signaling pathways. Food Chem Toxicol 50: 1734-1740, 2012.

23. Wu J, Wu X, Zhong D, et al: Short Hairpin RNA (shRNA) Ether à go-go 1 (Eag1) inhibition of human osteosarcoma angiogenesis via VEGF/PI3K/AKT signaling. Int J Mol Sci 13: 12573-12583, 2012.

24. van Maldegem AM, Bhosale A, Gelderblom HJ, Hogendoorn PC and Hassan AB: Comprehensive analysis of published phase I/II clinical trials between 1990-2010 in osteosarcoma and Ewing sarcoma confirms limited outcomes and need for translational investment. Clin Sarcoma Res 2: 5, 2012.

25. Ferrara N, Gerber HP and LeCouter J: The biology of VEGF and its receptors. Nat Med 9: 669-676, 2003. 